\title{
Resoluciones de disputas comerciales y desempeño económico regional en México
}

\section{Commercial Disputes Resolution and Regional Economic Performance in Mexico}

\author{
Juan Carlos Chávez \\ Felipe J. Fonseca \\ Manuel Gómez-Zaldívar
}

Recibido: 23 febrero 2016 /Aceptado: 24 agosto 2016

\section{Resumen}

En este trabajo, se analiza la relación existente entre el crecimiento económico y el estado de derecho en las entidades federativas de México, en el periodo 2006-2013. En particular, utilizamos información referente al tiempo que toma resolver disputas comerciales en cortes locales, como variable proxy para medir la eficiencia del sistema de justicia de las entidades. En principio, un menor tiempo en la resolución judicial de las disputas resultaría en un mayor crecimiento de las entidades donde las empresas se localizan; esto debido a que los procesos judiciales tienen costos, potencialmente importantes para las empresas, lo que repercute sobre el crecimiento de las mismas. Los resultados indican que una disminución de 100 días en el tiempo promedio que toma resolver disputas comerciales, estaría asociado con un incremento en la tasa de crecimiento del PIB per cápita de 0.6 por ciento en las entidades federativas.

Clasificación JEL: O43; O47.

Palabras Clave: Crecimiento Económico, Sistema de Justicia, Economías Regionales.

\footnotetext{
Dirección de Investigación Económica, Banco de México. Email: jcchavez@banxico.org.mx.

"*irección de Investigación Económica, Banco de México. Email: ffonseca@banxico.org.mx.

${ }_{* * * *}^{*}$ Autor para correspondencia. Dirección de Investigación Económica, Banco de México y Departamento de Economía y Finanzas de la Universidad de Guanajuato. Email: manuel.gomez@ugto.org. Dirección: Fraccionamiento 1, Col. El Establo S/N, C.P. 36250, Guanajuato, Guanajuato, México.
}

Las opiniones en este estudio corresponden a las de los autores y no necesariamente reflejan el punto de vista de Banco de México. 


\section{Abstract}

In this article, we analyze the relationship between the economic growth rate and a rule of law indicator in Mexican states during the period 2006-2013. Specifically, we employ information regarding the time it takes to solve commercial disputes in local courts, which we use as a proxy variable to measure the efficiency of the justice system. In principle, we expect that the shorter the time it takes to resolve commercial disputes, the higher the growth rates will be in the states where the firms are located. Long, drawn-out court disputes are costly for firms, which in turn may translate into lower growth rates due to the negative impact of these costs on their levels of investment. The results suggest that a 100-day decrease in the time it takes to resolve a commercial dispute is associated with an increase of 0.6 per cent in the average GDP per capita growth rate in Mexican states.

JEL Classification: O43; O47.

Keywords: Economic Growth, Justice System, Regional Economies.

"The inability of societies to
develop effective, low cost
enforcement is the most important
source of both historical and
contemporary underdevelopment
in the Third World."

Douglass North (1990).

\section{Introducción}

Como parte de la agenda que se ocupa de analizar los factores que determinan las diferencias en desarrollo y crecimiento económico entre países, diversos estudios han encontrado evidencia empírica que vincula al estado de derecho con el crecimiento económico [véase Acemoglu et al. (2001), Acemoglu y Robinson (2008), Barro (1996a), Djankov et al. (2006), Haggard (2010), Knack y Keefer (1995), Mauro (1995), North (1990), Rodrik (2007), entre otros]. En esos estudios, el estado de derecho es una variable multidimensional que se aproxima regularmente con indicadores de derechos de propiedad, eficiencia e independencia del sistema judicial, tasas de criminalidad, combate a la corrupción, estabilidad política, etc. En línea con esta literatura, consideramos que una característica fundamental que 
limita el crecimiento de México como un todo, y el de sus entidades, es la insuficiencia del estado de derecho ${ }^{1}$.

No obstante el importante papel que la literatura atribuye al estado de derecho sobre el desempeño económico, existe un número escaso de estudios aplicados a México en los que se analice la relación que existe entre el crecimiento de los diversos estados del país y alguna de las variables que arriba se mencionan, y que generalmente son usadas en estudios internacionales como proxies del estado de derecho. Entre estos pocos trabajos aplicados a México, se puede mencionar el de Weis y Rosenblatt (2010). Ellos analizan la relación entre el crecimiento en las entidades y factores relacionados con la transparencia gubernamental, como una vía de analizar determinantes institucionales del crecimiento regional, con datos para el periodo 2001-2005, y no encuentran evidencia de la misma. Sin embargo, sí encuentran que una medida de corrupción está negativamente asociada con el PIB per cápita estatal.

Enamorado et al. (2013), siguiendo esta misma literatura, estudian la relación entre crecimiento económico y crimen usando una metodología similar, pero empleando datos municipales para el periodo 2005-2010. En particular, ellos usan datos de ingreso, registros administrativos sobre crimen y violencia, y diversas medidas de gasto público. Sus resultados muestran que los crímenes relacionados con tráfico de drogas, sí tienen una relación negativa y significativa con el crecimiento del ingreso en los municipios; pero no existe evidencia de efecto negativo de los crímenes no relacionados con las drogas, sobre el crecimiento.

Adicionalmente, en su estudio sobre la convergencia en regiones mexicanas, Chiquiar (2005) encuentra un efecto nulo de las tasa de crimen sobre el crecimiento económico, tanto en el período 1970-1985 (convergencia) como en el período 1985-2001 (divergencia).

En este trabajo se analiza la relación entre el crecimiento económico, medido por el crecimiento del Producto Interno Bruto (PIB) per cápita, y un indicador de la rapidez de impartición de justicia en las entidades federativas de México. Consideramos que, en línea con lo argumentado por North (1990), la eficiencia y celeridad del sistema judicial para hacer cumplir contratos y hacer valer los derechos de propiedad son determinantes fundamentales del crecimiento económico de las diversas entidades del país. La lentitud del sistema judicial, o los procesos judiciales largos, tiene un

\footnotetext{
${ }^{1}$ Estudios existentes vinculan normalmente las bajas tasas de crecimiento observadas en las regiones del país con factores de índole económico, véase por ejemplo, Chiquiar (2005) y Hanson (2010).
} 
impacto negativo sobre la inversión que las empresas pueden realizar, ya que estos incrementan sus costos de transacción, limitando su liquidez disponible $\mathrm{y}$, por lo tanto, se traducen en menores niveles de inversión (véase por ejemplo Esposito et al. 2014, para el caso Italiano) que provocan, en el agregado, un efecto adverso sobre el desempeño y la productividad de las regiones donde estas se localizan (Ahsan, 2013) ${ }^{2}$. En este sentido, nuestro trabajo busca contribuir con la literatura sobre el tema, para el caso mexicano, utilizando una medida sobre la eficiencia del sistema judicial para resolver disputas comerciales en cortes locales.

Las estimaciones empíricas empleadas consisten en lo que se conoce como regresiones de crecimiento tipo Barro (1991, 1996a), metodología que regularmente se encuentra en esta literatura, y que ha sido ampliamente usada en trabajos que analizan datos en el nivel internacional (véase, por ejemplo, Mauro, 1995 y Djankov, 2006).

En particular, el trabajo empírico que aquí se presenta fue posible gracias a la disponibilidad de una medida relativamente nueva que reporta el Banco Mundial, referente al tiempo que en promedio tardan las resoluciones judiciales sobre disputas comerciales entre empresas en las distintas entidades federativas de México. Esta variable, hasta donde sabemos, no ha sido empleada por otro estudio en el caso mexicano (aunque sí en estudios de nivel internacional) ${ }^{3}$, y la misma está disponible para todas las entidades en los reportes de "Doing Business" del Banco Mundial de los años 2007, 2009, 2012 y $2014^{4}$.

Nuestros resultados muestran que la tasa de crecimiento del PIB per cápita de las entidades federativas, tiene una relación negativa y significativa con el número de días que toma resolver disputas comerciales. En particular, obtenemos que, una disminución de 100 días en el tiempo promedio de la resolución de disputas comerciales se asocia a un incremento de la tasa de

\footnotetext{
${ }^{2} \mathrm{Si}$ los fallos judiciales toman demasiado tiempo, estos tendrían impacto inmediato sobre la operación normal de una empresa, ya que estas podrían no disponer de capital suficiente para financiar sus operaciones normales o invertir en nuevos proyectos de desarrollo. Asimismo, la ausencia de confianza en el poder judicial podría hacer que las empresas se limitaran a hacer negocios con proveedores o clientes de confianza, ya conocidos, lo que reduciría la probabilidad de encontrar mejores oportunidades de negocios con empresas nuevas.

${ }^{3}$ Djankov et al., utilizan algunas otras de las medidas que también se reportan en la Base de Datos del Banco Mundial, Doing Business, para analizar la relación entre crecimiento económico y las regulaciones que rigen las actividades comerciales en 135 diferentes países. Para ello, también se emplea una metodología muy similar a la nuestra, basada en Barro (1991, 1996a) y Mauro (1995).

${ }^{4}$ Los datos presentados en los reportes de cada año corresponden a los del año inmediato anterior.
} 
crecimiento promedio anual del PIB per cápita, en las entidades, de 0.6 por ciento $\mathrm{y}$, como se verá en la sección correspondiente, las estimaciones son robustas a diferentes tipos de especificaciones. No obstante lo anterior, reconocemos que nuestros resultados se refieren a una medida específica del sistema de justicia y no implican necesariamente que sean generalizables a la noción más amplia de estado de derecho.

El resto del trabajo está organizado de la siguiente manera: en la Sección 1 se presentan los datos, se explica la metodología y se discuten los resultados obtenidos. Por último, en la Sección 2 se esbozan los comentarios finales.

\section{Datos, metodología y resultados}

Para analizar la relación existente entre desempeño económico y el tiempo de resolución de disputas comerciales, estimamos regresiones de crecimiento del tipo de Barro (1991,1996b), Mauro (1995) o Djankov (2006). Siguiendo a Chiquiar (2005), como medida del desempeño económico, se toma la tasa de crecimiento del PIB estatal, excluyendo la actividad petrolera para el período 2006-2013, mismo que se divide entre la población del estado correspondiente, para así obtener el PIB per cápita. La información del PIB y los datos de población se obtuvieron del sistema de cuentas nacionales del Instituto Nacional de Estadística y Geografía (INEGI) y del Consejo Nacional de Población (CONAPO), respectivamente. Los datos del tiempo de resolución de disputas comerciales se obtuvieron de la página de Doing Business del Banco Mundial ${ }^{5}$. El anexo 1, al final del documento, muestra que existe una gran heterogeneidad, entre las diversas entidades, en términos de todas las variables empleadas en el análisis empírico.

La gráfica 1 ilustra la relación entre tasa de crecimiento promedio de las entidades y el tiempo promedio que tomó resolver conflictos comerciales en ellas, en el periodo 2006-2013. Se puede observar una clara relación negativa y significativa entre estas dos variables ${ }^{6}$; lo cual es evidencia a favor de lo argumentado por North (1990), quien afirma que un sistema judicial eficaz en hacer cumplir contratos, es un determinante fundamental del desempeño económico.

Los modelos estimados se muestran en las ecuaciones 1 y 2 . Estos son similares a los empleados en los estudios que tratan de identificar los determinantes del crecimiento económico, utilizando datos de corte

\footnotetext{
${ }^{5}$ Los datos están disponibles en www.doingbusiness.org.

${ }^{6}$ La estimación de la relación lineal entre las dos variables se muestra en la gráfica 1. El estadístico entre paréntesis corresponde al error estándar robusto de la variable $\mathrm{X}$, tiempo promedio de resolución de los conflictos comerciales.
} 
transversal. La diferencia principal entre ambos radica en que la ecuación 1 controla el impacto del tiempo de resolución de disputas comerciales por un índice (ICP), que captura el nivel de desarrollo de cada entidad del país. Esta variable incluye indicadores de infraestructura urbana, variables demográficas, variables financieras e infraestructura de comunicaciones y transporte, y fue construida a través del método de componentes principales? En tanto, la ecuación 2 considera variables de control "estándar" en la literatura de crecimiento (véase Chiquiar, 2005; Rodríguez-Oreggia, 2005). Además, ambos modelos también incluyen como control el PIB per cápita, al inicio del periodo.

\section{Gráfica 1}

Tasa de crecimiento y tiempo que toma resolver una disputa comercial, 2006-2013

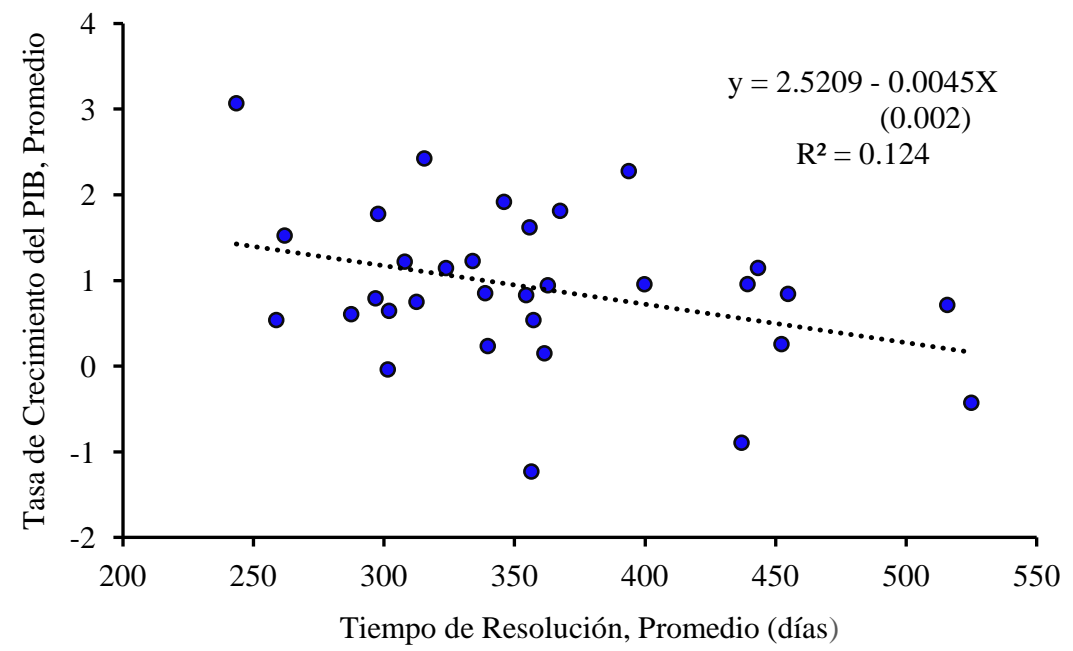

Fuente: Estimaciones propias con datos de INEGI, Banco Mundial y CONAPO.

$\gamma_{i}=\alpha+\ln \left(P I B_{i 0}\right)+\theta \cdot T_{i}+\delta \cdot I C P_{i}+\varepsilon_{i}$

$\gamma_{i}=\alpha+\ln \left(P I B_{i 0}\right)+\theta \cdot T_{i}+\gamma \cdot$ Infraestructura $_{i}+\vartheta \cdot H K_{i}+\epsilon_{i} ;$

donde $\gamma_{i}$ es la tasa de crecimiento promedio anual de PIB per cápita, en el periodo de estudio, de cada una de las entidades; $P I B_{i 0}$ es el PIB per cápita de las entidades federativas en el periodo inicial; $T_{i}$ es el tiempo promedio, días, que tomó resolver las disputas; en tanto, ICP es el índice de componentes principales anteriormente referido; infraestructura se refiere a

\footnotetext{
${ }^{7}$ El anexo 2 presenta los detalles de la construcción de este índice, que se obtuvo de Chávez y López (2013).
} 
la densidad telefónica en las entidades federativas (definida como el número de líneas por cada 100 habitantes); mientras que $H K$, corresponde al grado promedio de escolaridad de la población de 15 años y más, en las entidades federativas. Para ambas variables, la fuente de información es el INEGI.

Tabla 1

Resultados de las estimaciones $^{1 /}$

\begin{tabular}{|c|c|c|c|c|}
\hline Variables & \multicolumn{2}{|c|}{$\begin{array}{l}\text { Regresiones de Crecimiento } \\
\text { Ecuación } 1 \\
\end{array}$} & \multicolumn{2}{|c|}{$\begin{array}{c}\text { Regresiones de Convergencia } \\
\text { Ecuación } 2 \\
\end{array}$} \\
\hline \multirow{2}{*}{ Intercepto } & 2.380 & 3.257 & 3.829 & 5.224 \\
\hline & (2.94) & (3.09) & (1.53) & (2.01) \\
\hline \multirow{2}{*}{ Tiempo } & -0.004 & -0.007 & -0.004 & -0.006 \\
\hline & $(-2.18)$ & $(-2.43)$ & $(-2.16)$ & $(-2.60)$ \\
\hline \multirow{2}{*}{ ICP } & 0.226 & 0.273 & 0.303 & 0.380 \\
\hline & (1.49) & (1.70) & (1.36) & (1.68) \\
\hline \multirow{2}{*}{$\llbracket P I B \beth$} & - & $-\ldots$ & -0.343 & -0.486 \\
\hline & & & $(-0.63)$ & $(-0.93)$ \\
\hline$R^{\wedge i}$ & 0.18 & ------ & 0.19 & ----- \\
\hline Ramsey-Reset & 0.46 & ------ & 0.54 & ------ \\
\hline Normalidad & 0.67 & ------ & 0.93 & ----- \\
\hline Hausman & ----- & 0.05 & ---- & 0.03 \\
\hline Sargan & ----- & 0.19 & ----- & 0.38 \\
\hline $\begin{array}{l}\text { Significancia } \\
\text { conjunta } F\end{array}$ & ----- & 18.37 & ----- & 22.52 \\
\hline Método & MCO & MC2E & MCO & MC2E \\
\hline
\end{tabular}

Fuente: Estimaciones propias con datos de INEGI, Banco Mundial y CONAPO. 1/ Los estadísticos $t$ se muestran entre paréntesis. Los errores estándar se calculan usando el método de White. Los valores que acompañan a las pruebas de especificación RamseyReset, Normalidad (Jarque-Bera), Hausman y Sargan son p-values; MCO y MC2E se refieren a las estimaciones por Mínimos Cuadrados Ordinarios y por Mínimos Cuadrados en dos Etapas.

Dado que en esta literatura generalmente se señala la potencial existencia de un problema de endogeneidad, esto es, que el crecimiento económico y la calidad de los instituciones sean variables que evolucionan conjuntamente y se retroalimenten, en consecuencia, la estimación de los modelos propuestos también se lleva a cabo utilizando variables instrumentales a través del método de estimación de mínimos cuadrados en dos etapas (MC2E). Para ello, instrumentamos el tiempo de resolución de las disputas comerciales usando dos variables. La primera es un índice de fraccionalización etnolongüistica (IFE) ${ }^{8}$, como el empleado por Mauro (1995). Siguiendo a este

\footnotetext{
${ }^{8}$ Este índice se define de la siguiente manera: $\mathrm{IFE}=1-\sum_{\mathrm{i}=1}^{\mathrm{I}}\left(\mathrm{n}_{\mathrm{i}} / \mathrm{N}\right)^{2}, \mathrm{i}=1, \ldots, \mathrm{I}$; donde $n_{i}$ es el número de personas de cada grupo etnolingüístico, $N$ es la población estatal total, mientras que $I$ es el número total de grupos etnolingüísticos en cada entidad. De tal manera que, mientras más grande sea el índice IFE mayor fragmentación etnolingüística existirá.
} 
autor, la justificación del uso de esta variable como instrumento, se debe a que la presencia de muchos grupos etnolingüísticos se asocia con mayores niveles de corrupción, debido - por ejemplo- a que las burocracias tienden a favorecer a miembros de su mismo grupo etnolingüístico. La segunda es el valor de la variable del tiempo para resolver disputas comerciales en el período inicial, tal y como se lleva a cabo en Barro (1996a), donde algunas de las variables instrumentales que se utilizan son los valores iniciales de los regresores. En su estudio, Barro menciona que este enfoque es adecuado debido a que los residuales de las estimaciones de las ecuaciones de crecimiento exhiben poca correlación con los valores iniciales de las variables, situación que se verifica en nuestros resultados.

Adicionalmente, los instrumentos antes mencionados se relacionan positiva y significativamente con la variable tiempo promedio de resolución de disputas comerciales $\left(T_{i}\right)$, y no tienen relación con el término de error de las ecuaciones estructurales (1) y (2), por lo que cumplen con los requisitos necesarios para dicho fin (Wooldridge, 2009) ${ }^{9}$.

El cuadro 1 presenta los resultados. Las pruebas de diagnóstico para las estimaciones de variables instrumentales (MC2E) indican que el estadístico de la prueba de Hausman rechaza, en ambos casos, la hipótesis nula de exogeneidad, por lo que hay evidencia de que la estimación mediante MC2E es preferible respecto a la de MCO. En tanto, la prueba de Sargan señala que no es posible rechazar la hipótesis nula de que los instrumentos considerados son válidos ${ }^{10}$. Los resultados de las regresiones muestran que la relación ilustrada en la gráfica 1 es robusta, tanto del parámetro estimado como de su significancia estadística, ya que se mantienen cuando se incluyen los controles adicionales. Las estimaciones $\mathrm{MC} 2 \mathrm{E}$ muestran que el parámetro estimado asociado a la variable de interés, tiempo de resolución, es de -0.006; en tanto que las de MCO, el rango es de -0.003 a -0.004. Esto implica que las estimaciones obtenidas por MCO, al ser sesgadas, subestiman el impacto que la pronta impartición de justicia tiene sobre el crecimiento. Las estimaciones puntuales implican que una reducción de 100 días en el tiempo promedio de resolución de disputas comerciales, se asocia a un incremento de la tasa de crecimiento anual del PIB per cápita, de las entidades, de 0.6 por ciento. Con

Para una explicación más amplia del uso de IFE como variable instrumental (véase Mauro, 1995).

${ }^{9} \mathrm{Se}$ corrieron regresiones por MCO para cada instrumento y la variable $T_{i}$, se encuentra una relación positiva y significativa, también se verifica que los residuales de las regresiones estructurales no tienen relación con los instrumentos empleados.

${ }^{10}$ Adicionalmente, la prueba $\mathrm{F}$ de significancia conjunta de la primera etapa, de MC2E, resulta significativa, por encima del valor de 10 (que generalmente se usa como regla de dedo), lo que indica la relevancia del poder explicativo de los instrumentos empleados. 
un intervalo de confianza del $95 \%$, podemos asegurar que dicho incremento está situado entre 0.4 y 0.8 por ciento del PIB per cápita.

Respecto de las variables de control, de acuerdo con la ecuación (1), el parámetro que acompaña a la variable que mide el desarrollo de las entidades, ICP, es siempre estimado con el signo correcto (positivo), aunque solo marginalmente significativo al $10 \%$ en uno de los casos. Tal y como la teoría sugiere, las tasas de crecimiento están positivamente relacionadas con las entidades que tienen mayor provisión de infraestructura en comunicaciones y transporte, financiera, urbana y mejores indicadores demográficos. Por su parte, los controles empleados en la ecuación (2) estiman un impacto positivo y significativo de la variable de infraestructura sobre el crecimiento económico. Con respecto a la variable de capital humano empleada, esta tiene el signo contrario al esperado, aunque no es estadísticamente significativa ${ }^{11}$.

El parámetro que acompaña a la variable PIB inicial es en todos los casos estimado negativo, aunque no significativo, lo que está en línea con estudios aplicados a México, que no encuentran evidencia sobre convergencia para el período posterior a 1985 (Chiquiar, 2005).

Respecto de los estadísticos de diagnóstico, la prueba de Ramsey señala que las estimaciones consideradas están correctamente especificadas, al tiempo que tampoco podemos rechazar la hipótesis de normalidad en los residuales. Finalmente, con el propósito de descartar algún proceso de dependencia espacial en las estimaciones, se aplicaron pruebas para detectar la presencia de este tipo de efectos a las estimaciones de las ecuaciones (1) y (2). De encontrar evidencia en este sentido, estaríamos en presencia de estimaciones sesgadas e ineficientes (LeSage y Pace, 2009). Definiendo una matriz de dependencia espacial con base en el criterio de contigüidad entre entidades federativas, los resultados que se muestran en el anexo 3 señalan que no existe presencia de este tipo de efectos, tanto en el término de error (spatial error) como en la variable dependiente (spatial lag).

\footnotetext{
${ }^{11}$ Se consideró el porcentaje de población con educación media y superior sobre la población de 25 años y más, a partir de información de la ENOE; no obstante, los resultados no fueron distintos, en términos de la dirección y signo del coeficiente. Cabe mencionar que estudios sobre determinantes del crecimiento, tales como el de Caselli et al. (1996), y Forbes (2000) han encontrado resultados similares respecto del impacto de esta variable sobre el crecimiento.
} 


\section{Comentarios finales}

El estado de derecho como determinante del crecimiento económico es un tema que ha sido ampliamente discutido teóricamente, y contrastado empíricamente en múltiples trabajos en el nivel internacional. Estudios previos aplicados a México no han conseguido mostrar este tipo de evidencia de una manera clara y robusta. Las estimaciones presentadas en este trabajo resaltan la importancia que las instituciones, particularmente la eficiencia en la impartición de justicia, tienen para el crecimiento económico en las entidades de nuestro país.

Existen grandes diferencias, entre entidades federativas, en cuanto al tiempo que tardan en la resolución de disputas comerciales. Esto sugiere que hay un ámbito para la implementación de mejoras en términos de política pública para alcanzar un mayor crecimiento económico. A este respecto, la figura de los Juicios Orales Mercantiles, por ejemplo ${ }^{12}$, constituyen un paso hacia adelante, y contribuirán a mejorar las condiciones para alcanzar mayores tasas de crecimiento en las diferentes regiones del país, la magnitud de este efecto positivo dependerá de la eficacia con que sean implementados.

\section{Referencias}

[1] Acemoglu D., Johnson, S. y Robinson, J. (2001). "The Colonial Origins of Comparative Development: an Empirical Investigation." The American Economic Review, 91(5), 1369-1401.

[2] Acemoglu D., y Robinson, J. (2008). "The Role of Institutions in Growth and Development." Working Paper 10, Commission on Growth and Development, The World Bank.

[3] Ahsan, R. N. (2013). "Input Tariffs, Speed of Contract Enforcement, and the Productivity of Firms in India." Journal of International Economics, 90(1), 181-192.

[4] Banco Mundial (2007, 2009, 2012, 2014). "Doing Business in Mexico". Banco Mundial, disponible en: www.doingbusiness.org

[5] Barro, R. (1991), "Economic Growth in a Cross Section of Countries", Quarterly Journal of Economics, 106(2), 407-443.

[6] Barro, R. (1996a), "Democracy and Growth", Journal of Economic Growth, 1(1), $1-27$.

[7] Barro, R. (1996b). "Determinants of Economic Growth: A Cross-Country Empirical Study." NBER, Working Paper No. 5698.

\footnotetext{
${ }^{12}$ De acuerdo con la Comisión Federal de Mejora Regulatoria (COFEMER), se estima que es posible que el plazo que transcurre desde que es presentada la demanda hasta el dictado de sentencia se reduzca hasta en 112 días, disminuyendo con ello alrededor de una cuarta parte la duración actual promedio de un juicio en materia mercantil.
} 
[8] Caselli, F., Esquivel, G., y Lefort, F. (1996). "Reopening the Convergence Debate: a New Look at Cross-Country Growth Empirics". Journal of Economic Growth, 1(3), 363-389.

[9] Chávez, Juan C. y López, L. F. (2013), "Diferencias en la Productividad del Trabajo de la Industria Manufacturera: ¿Cómo se Relacionan con la Provisión de Infraestructura?", Mimeo, Banco de México.

[10] Chiquiar, D. (2005). "Why Mexico's Regional Income Converge Broke Down". Journal of Development Economics, 77, 257-275.

[11] Djankov, Simeon, Mcliesh, C. y Ramalho, R. M. (2006). "Regulation and Growth". Economics Letters, 92, 395-401.

[12] Enamorado, T., López-Calva, L. y Rodríguez-Castelan, C. (2013). "Crime and Growth Convergence, Evidence from Mexico". The World Bank, Latin America and Caribbean Region, Poverty Reduction and Economic Management Unit, Policy Research Working Paper No. 6730.

[13] Esposito, G., Lanau, S. y Pompe, S. (2014). "Judicial System Reform in Italy: a Key to Growth.” International Monetary Fund, Working Paper No. 14/32.

[14] Forbes, K. J. (2000). "A Reassessment of the Relationship between Inequality and Growth.” American Economic Review, 90(4), 869-887.

[15] Hotelling, H. (1933). "Analysis of a Complex of Statistical Variables into Principal Components”. Journal of Educational Psychology, 24(6), 417441.

[16] Jolliffe, I. (2002). Principal Component Analysis. John Wiley \& Sons, Ltd.

[17] Haggard, S. y Tiede, L. (2010). "The Rule of Law and Economic Growth: Where are We?" Artículo preparado para presentarse en la Conferencia Measuring the Rule of Law, 2010, UT School of Law.

[18] Hanson, G. (2010). "Why isn't Mexico Rich?" Journal of Economic Literature, 48(4), 987-1004.

[19] Knack, S., y Keefer, P. (1995). "Institutions and Economic Performance: CrossCountry Test Using Alternative Institutional Measures." Economic and Politics, 7(3), 207-227.

[20] LeSage, J. P., y Pace, R. K. (2009). Introduction to Spatial Econometrics, CRC Press.

[21] Mauro, P. (1995). "Corruption and Growth". The Quarterly Journal of Economics, 110(3), 681-712.

[22] North, D. (1990). Institutions, Institutional Change, and Economic Performance. New York: Cambridge University Press.

[23] Pearson, K. (1901). "On Lines and Planes of Closest Fit to Systems of Points in Space”. Philosophical Magazine, 2(11), 559-572.

[24] Rodrik D. (2007). "Institutions for High Quality Growth: What are They and How to Acquire Them". Studies in Comparative International Development, 35(3), 3-31.

[25] Rodríguez-Oreggia, E. (2005). "Regional Disparities and Determinants of Growth in Mexico". The Annals of Regional Science, 39(2), 207-220.

[26] Weiss, E., y D. Rosenblatt (2010). "Regional Economic Growth in Mexico, Recent Evolution and the Role of Governance." The World Bank, Latin America and Caribbean Region, Agriculture and Rural Development Unit, Policy Research Working Paper No. 5369.

[27] Wooldridge (2009). Introductory Econometrics. A Modern Approach, Thomson. 
Anexo 1

Tabla 2

Estadísticos descriptivos de las variables empleadas

\begin{tabular}{|c|c|c|c|c|c|c|c|}
\hline & \multicolumn{5}{|c|}{ Tiempo de resolución de Disputas (número de días) } & \multirow{2}{*}{$\begin{array}{l}\text { Crecimiento } \\
\text { Económico }^{1 /}\end{array}$} & \multirow{2}{*}{$\begin{array}{c}\text { Índice de } \\
\text { Infraestructura }^{2}\end{array}$} \\
\hline & 2007 & 2009 & 2012 & 2014 & Promedio & & \\
\hline Aguascalientes & 290 & 327 & 271 & 303 & 298 & 1.78 & 1.70 \\
\hline Baja California & 470 & 446 & 416 & 416 & 437 & -0.89 & 0.90 \\
\hline Baja California Sur & 581 & 497 & 497 & 525 & 525 & -0.43 & 0.60 \\
\hline Campeche & 430 & 361 & 355 & 280 & 357 & -1.23 & 0.20 \\
\hline Coahuila & 422 & 270 & 270 & 270 & 308 & 1.22 & 0.90 \\
\hline Colima & 245 & 343 & 310 & 310 & 302 & 0.65 & 2.90 \\
\hline Chiapas & 386 & 386 & 337 & 337 & 362 & 0.15 & -0.80 \\
\hline Chihuahua & 280 & 290 & 290 & 290 & 288 & 0.61 & 0.60 \\
\hline Distrito Federal & 345 & 415 & 415 & 400 & 394 & 2.28 & 3.50 \\
\hline Durango & 305 & 259 & 243 & 228 & 259 & 0.54 & 0.20 \\
\hline Guanajuato & 326 & 385 & 385 & 288 & 346 & 1.92 & 0.90 \\
\hline Guerrero & 304 & 375 & 375 & 375 & 357 & 0.54 & -0.90 \\
\hline Hidalgo & 280 & 330 & 320 & 320 & 313 & 0.75 & 0.50 \\
\hline Jalisco & 275 & 360 & 360 & 360 & 339 & 0.85 & 1.10 \\
\hline México & 318 & 375 & 375 & 350 & 355 & 0.83 & 1.60 \\
\hline Michoacán & 381 & 390 & 340 & 340 & 363 & 0.95 & 0.40 \\
\hline Morelos & 390 & 461 & 461 & 461 & 443 & 1.15 & 1.80 \\
\hline Nayarit & 429 & 310 & 310 & 310 & 340 & 0.24 & 0.60 \\
\hline Nuevo León & 320 & 256 & 236 & 236 & 262 & 1.53 & 1.40 \\
\hline Oaxaca & 330 & 300 & 353 & 353 & 334 & 1.23 & -1.00 \\
\hline Puebla & 445 & 391 & 391 & 372 & 400 & 0.96 & 0.50 \\
\hline Querétaro & 290 & 324 & 324 & 324 & 316 & 2.43 & 1.10 \\
\hline Quintana Roo & 568 & 560 & 560 & 375 & 516 & 0.72 & 0.60 \\
\hline San Luis Potosí & 360 & 381 & 341 & 341 & 356 & 1.62 & 0.10 \\
\hline Sinaloa & 337 & 290 & 290 & 270 & 297 & 0.79 & 0.80 \\
\hline Sonora & 372 & 366 & 366 & 366 & 368 & 1.81 & 0.70 \\
\hline Tabasco & 353 & 314 & 314 & 314 & 324 & 1.15 & 0.40 \\
\hline Tamaulipas & 415 & 301 & 245 & 245 & 302 & -0.04 & 0.80 \\
\hline Tlaxcala & 415 & 484 & 455 & 455 & 452 & 0.26 & 1.90 \\
\hline Veracruz & 470 & 382 & 470 & 435 & 439 & 0.96 & 0.10 \\
\hline Yucatán & 495 & 428 & 398 & 498 & 455 & 0.84 & 0.50 \\
\hline Zacatecas & 230 & 248 & 248 & 248 & 244 & 3.07 & 0.40 \\
\hline Promedio & 371 & 363 & 354 & 344 & 358 & 0.91 & 0.78 \\
\hline Desviación estándar & 86.6 & 74.2 & 78.4 & 74.9 & 70.5 & 0.90 & 0.94 \\
\hline Mínimo & 230 & 248 & 236 & 228 & 244 & -1.23 & -1.00 \\
\hline Máximo & 581 & 560 & 560 & 525 & 525 & 3.07 & 3.50 \\
\hline
\end{tabular}

Nota: 1/ Tasa de crecimiento promedio anual del PIB per cápita, excluyendo la actividad petrolera. 2/ El índice se presenta en forma estandarizada y se obtuvo de Chávez y López (2013). Fuente: Banco Mundial (reporte Doing Business, varios años) y estimaciones propias con datos de INEGI y del reporte Doing Business del Banco Mundial. 


\section{Anexo 2}

\section{Índice de infraestructura de las entidades, construido usando el método de componentes principales.}

El empleo del análisis de componentes principales es comúnmente utilizado cuando se trabaja con bases de datos que contienen un número considerable de variables interrelacionadas, y es deseable reducir la dimensión de estas, y a la vez conservar la mayoría de la variación presentada en los datos originales. Esta técnica fue desarrollada a partir de los trabajos de Person (1901) y posteriormente de Hotelling (1933). Como se mencionó, el objetivo de este procedimiento es describir la varianza de un conjunto de datos por medio de una transformación de variables en un nuevo conjunto con mayor parsimonia. Para lograr esto, se construye un conjunto de variables no correlacionadas, donde cada una de estas es una combinación lineal de los datos originales. Para mayores detalles técnicos se puede consultar la obra de Jolliffe (2002).

Para el presente trabajo se construyó una base de datos con trece variables con información promedio de distintos tipos de infraestructura de los años 2000 al 2010. Dichas variables se presentan en el siguiente cuadro.

Tabla 3

Variables incluidas en la construcción del índice de infraestructura

\begin{tabular}{|c|c|c|}
\hline Infraestructura & Variable & Fuente \\
\hline \multicolumn{3}{|l|}{ Comunicaciones } \\
\hline \multicolumn{3}{|l|}{ y Transportes } \\
\hline Carreteras & $\begin{array}{l}\text { Longitud de carreteras pavimentadas / } \\
\text { Superficie de la entidad }\end{array}$ & INEGI \\
\hline Ferrocarriles & Longitud de vías férreas/superficie de la entidad & INEGI \\
\hline Puertos & Longitud de atraque/superficie de la entidad & INEGI \\
\hline Aeropuertos & Carga (en kilogramos) per cápita & INEGI \\
\hline Telefonía & Número de líneas per cápita & INEGI \\
\hline \multicolumn{3}{|l|}{ Urbana } \\
\hline Electricidad & Proporción de hogares con electricidad & INEGI \\
\hline Drenaje & Proporción de hogares con drenaje & INEGI \\
\hline Agua & Proporción de hogares con agua potable & INEGI \\
\hline \multicolumn{3}{|c|}{ T } \\
\hline Educación & $\begin{array}{l}\text { Grado promedio de escolaridad } \\
\text { (población de } 15 \text { años y más) }\end{array}$ & INEGI \\
\hline Salud & Esperanza de vida & CONAPO \\
\hline \multicolumn{3}{|l|}{ Financiera } \\
\hline Sucursales bancarias & Número de sucursales per cápita & CNBV \\
\hline Crédito & Crédito/PIB de la entidad & Banxico, INEGI \\
\hline Crédito Industrial & Crédito industrial / Unidades manufactureras & Banxico, INEGI \\
\hline
\end{tabular}

Fuente: Chávez y López (2013). 
En la tabla 4, se observan los componentes principales para las variables de interés. En la segunda columna se muestra el valor propio de cada componente, es decir, la varianza asociada a cada uno, la tercera columna corresponde a la proporción de la varianza explicada, mientras que la cuarta columna ilustra los valores correspondientes a la $R^{2}$ acumulada. El criterio para elegir el número de componentes principales que se utilizaron para construir el índice global, consistió en tomar el número de componentes necesarios hasta lograr una $R^{2}$ acumulada al menos de 0.90. Bajo este precepto, en el cálculo del índice global, se tomaron en cuenta los primeros cinco componentes del análisis, los cuales explican el 91 por ciento de la varianza total de los datos.

Tabla 4

Análisis de componentes principales

Componentes Valores propios Proporción R2 acumulada

\begin{tabular}{lllr}
\hline Componente 1 & 7.10 & 0.55 & 0.55 \\
Componente 2 & 2.03 & 0.16 & 0.7 \\
Componente 3 & 1.31 & 0.10 & 0.8 \\
Componente 4 & 0.94 & 0.07 & 0.87 \\
Componente 5 & 0.43 & 0.03 & 0.91 \\
Componente 6 & 0.30 & 0.02 & 0.93 \\
Componente 7 & 0.27 & 0.02 & 0.95 \\
Componente 8 & 0.20 & 0.02 & 0.97 \\
Componente 9 & 0.15 & 0.01 & 0.98 \\
Componente 10 & 0.11 & 0.01 & 0.99 \\
Componente 11 & 0.08 & 0.01 & 0.99 \\
Componente 12 & 0.05 & 0.00 & 1.00 \\
Componente 13 & 0.04 & 0.00 & 1.00 \\
\hline
\end{tabular}

Fuente: Chávez y López (2013). 


\section{Anexo 3}

Resultado de las pruebas de dependencia espacial aplicadas a las estimaciones de las ecuaciones (1) y (2)

a) Estimaciones Ecuación (1)

\begin{tabular}{|c|c|c|c|}
\hline Test & Statistic & $\mathrm{df}$ & $\mathrm{p}$-value \\
\hline \multicolumn{4}{|l|}{ Spatial error: } \\
\hline Moran's I & 0.183 & 1 & 0.855 \\
\hline Lagrange multiplier & 0.083 & 1 & 0.773 \\
\hline Robust Lagrange multiplier & 2.583 & 1 & 0.108 \\
\hline \multicolumn{4}{|l|}{ Spatial lag: } \\
\hline Lagrange multiplier & 0.034 & 1 & 0.855 \\
\hline Robust Lagrange multiplier & 2.533 & 1 & 0.111 \\
\hline
\end{tabular}

Fuente: Estimaciones propias.

b) Estimaciones Ecuación (2)

\begin{tabular}{|c|c|c|c|}
\hline Test & Statistic & df & p-value \\
\hline \multicolumn{4}{|l|}{ Spatial error: } \\
\hline Moran's I & -0.472 & 1 & 1.363 \\
\hline Lagrange multiplier & 1.017 & 1 & 0.313 \\
\hline Robust Lagrange multiplier & 2.614 & 1 & 0.106 \\
\hline \multicolumn{4}{|l|}{ Spatial lag: } \\
\hline Lagrange multiplier & 0.17 & 1 & 0.68 \\
\hline Robust Lagrange multiplier & 1.767 & 1 & 0.184 \\
\hline
\end{tabular}

Fuente: Estimaciones propias. 